\title{
Exploration of Fibonacci function
}

\author{
Prof. K. Raja Rama Gandhi \\ Department of Mathematics, \\ BITS-Vizag
}

Email: rrmath28@gmail.com

Keywords: Fibonacci function, Golden ratio

ABSTRACT In this paper, I define Fibonacci function (probably unknown) on Real number field, for all $x \in R, F: R \rightarrow R, \ni F(x+n)=f_{n} F(x+1)+f_{n-1} F(x)$ Also, I defined the limit value of Fibonacci function, which is closed to $1.618 \ldots$ where $\mathrm{x}$ tends to infinity. Including, Fibonacci sum as well.

\section{INTRODUCTION}

Fibonacci numbers have been studied in many different forms for centuries and the literature on the subject is vast. One of the remarkable qualities of these numbers is the diversity of mathematical models where they play some sort of role and where their properties are of importance in explaining the ability of the model under discussion to explain whatever implications are characteristic in it. The fact that the ratio of successive Fibonacci numbers approaches the Golden ratio (section) [1] rather quickly as they go to infinity probably has a good deal to do with the observation made in the previous sentence. In this paper, I would like to introduce a Fibonacci functions (probably unknown to many) and its limit value in the second section. Also, I would like to discus some results on Fibonacci summation in the last section.

The reason for writing this paper is Fibonacci numbers are come from nature and we find these numbers everywhere in different forms in our daily life. I published a paper [4], which is described the identities of Fib-series. I just pasted some pic(s) with history, which will tell us the importance of Fibonacci series.
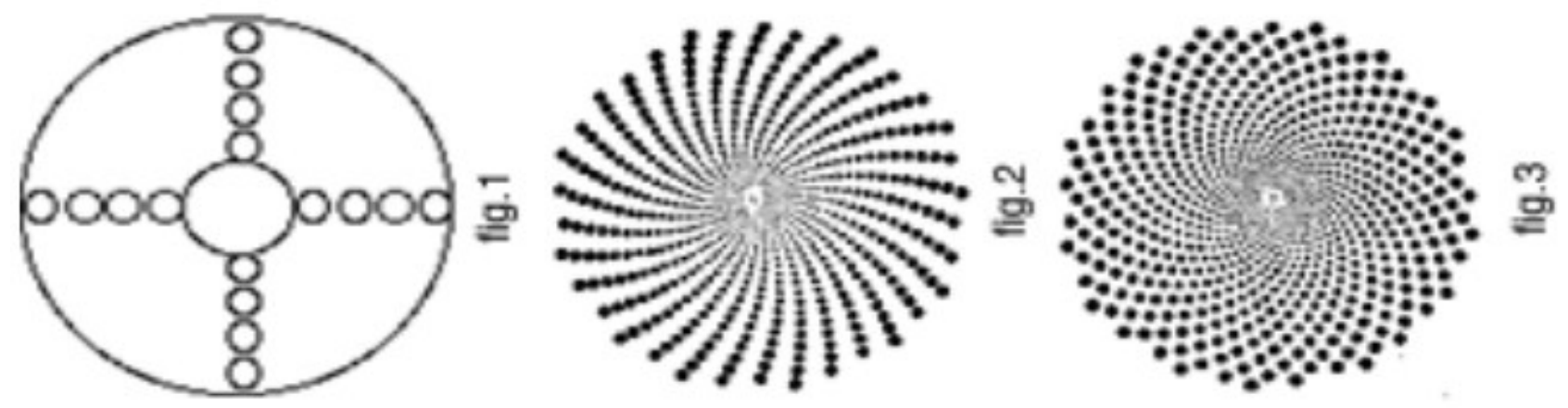

The Fibonacci sequence is named after Leonardo of Pisa, who was known as Fibonacci. Fibonacci's 1202 book Liber Abaci introduced the sequence to Western European mathematics, although the sequence had been described earlier in Indian mathematics. (By modern convention, the sequence begins with $\mathrm{F}_{0}=0$. The Liber Abaci began the sequence with $\mathrm{F}_{1}=1$, omitting the initial 0 and the sequence is still written this way by some.)

Fibonacci numbers are closely related to Lucas numbers in that they are a complementary pair of Lucas sequences. They are intimately connected with the golden ratio, for example the closest rational approximations to the ratio are $2 / 1,3 / 2,5 / 3,8 / 5, \ldots$. Applications include computer algorithms such as the Fibonacci search technique and the Fibonacci heap data structure, and graphs called Fibonacci cubes used for interconnecting parallel and distributed systems. They also 
appear in biological settings, such as branching in trees, phyllotaxis (the arrangement of leaves on a stem), the fruit spouts of a pineapple, the flowering of artichoke, an uncurling fern and the arrangement of a pine cone [2]
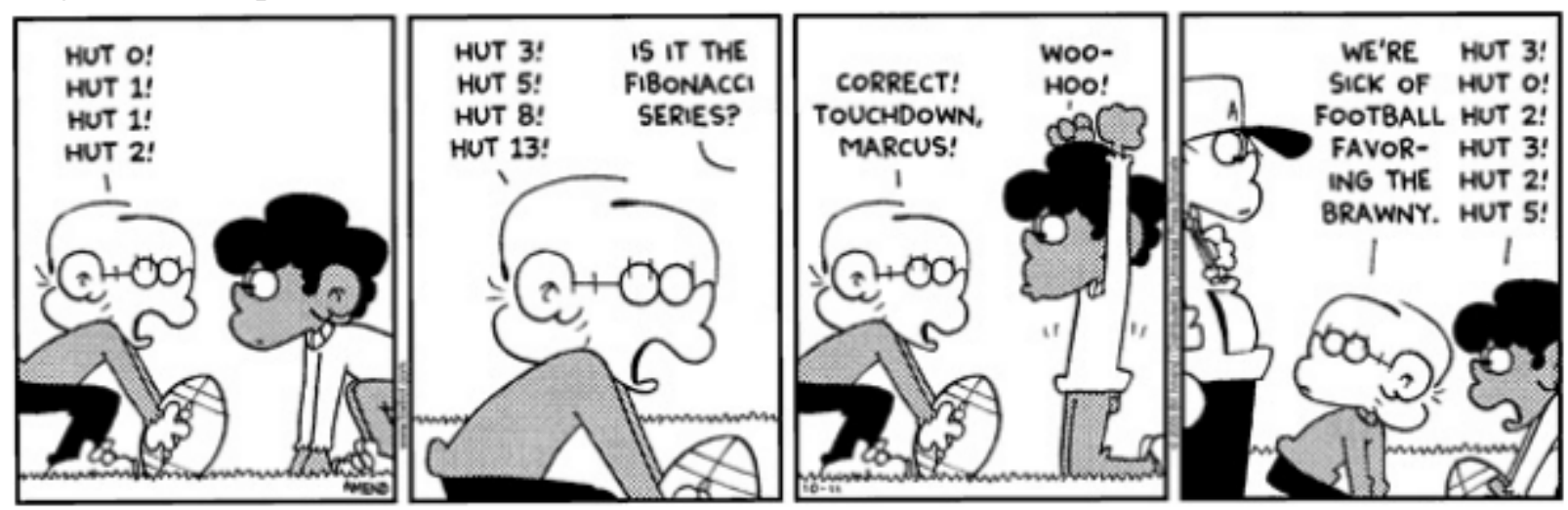

[3] The first 21 Fibonacci numbers $\mathrm{F}_{\mathrm{n}}$ for $\mathrm{n}=0,1,2, \ldots, 20$ are:

$$
\begin{aligned}
& \begin{array}{lllllllllll}
F_{0} F_{1} F_{2} F_{3} F_{4} F_{5} F_{6} F_{7} F_{8} F_{9} F_{10} F_{11} & F_{12} & F_{13} & F_{14} & F_{15} & F_{16} & F_{17} & F_{18} & F_{19} & F_{20}
\end{array}
\end{aligned}
$$

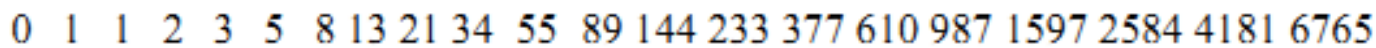

The sequence can also be extended to negative index $n$ using the re-arranged recurrence relation $F_{n-2}=F_{n}-F_{n-1}$,

which yields the sequence of "negafibonacci" numbers satisfying

$F_{-n}=(-1)^{n+1} F_{n}$.

Thus the bidirectional sequence is

$F_{-8} F_{-7} F_{-6} F_{-5} F_{-4} F_{-3} F_{-2} F_{-1} F_{0} F_{1} F_{2} F_{3} F_{4} F_{5} F_{6} F_{7} F_{8}$

$\begin{array}{llllllllllllllllll}-21 & 13 & -8 & 5 & -3 & 2 & -1 & 1 & 0 & 1 & 1 & 2 & 3 & 5 & 8 & 13 & 21\end{array}$

In this paper, I define Fibonacci function on Real number field, for all $x \in R, F: R \rightarrow R$, э $F(x+n)=f_{n} F(x+1)+f_{n-1} F(x)$, where $F(x)$ and $f_{n}$ are Fibonacci function and Fibonacci sequence respectively. Also, I defined the limit value of Fibonacci function, which is closed to $1.618 \ldots$ where $\mathrm{x}$ tends to infinity. Including, Fibonacci sum as well.

\section{FIBONACCI FUNCTION}

\subsection{Observation}

Let us consider $f_{n} \rightarrow$ Fibonacci sequence and $F(x) \rightarrow$ Fibonacci function. Let us fix $f_{0}=0$ and $f_{l}=1=f_{2}$ for $n \geq 2$, where $n \in R$. Now, we define for all $x \in R$,

$$
F(x+n)=f_{n} F(x+1)+f_{n-1} F(x)
$$

Proof: For $F(x)=\frac{\alpha^{x}-\beta^{x}}{\sqrt{5}}$ with $\alpha=\frac{1+\sqrt{5}}{2} \& \beta=\frac{1-\sqrt{5}}{2}$

From (1), we can observe that $\alpha \beta=-1$ and $\alpha+\frac{1}{\alpha}=\sqrt{5} \& \beta+\frac{1}{\beta}=-\sqrt{5}$.

Now, for all $n \geq 2$ with $n \in N$, 
We have $F(n)=f_{n}$

and we wish to conclude $(*)$

R.H.S. part of $(*)$ is same as

$=\frac{1}{5}\left[\left(\alpha^{n}-\beta^{n}\right)\left(\alpha^{x+1}-\beta^{x+1}\right)+\left(\alpha^{n-1}-\beta^{n-1}\right)\left(\alpha^{x}-\beta^{x}\right)\right]$

$=\frac{1}{5}\left[\alpha^{x+n+1}-\alpha^{n} \beta^{x+1}-\alpha^{x+1} \beta^{n}+\beta^{x+n+1}+\alpha^{x+n-1}-\alpha^{n-1} \beta^{x}-\alpha^{x} \beta^{n-1}+\beta^{x+n-1}\right]$

$=\frac{1}{5}\left[\alpha^{x+n}\left(\alpha+\frac{1}{\alpha}\right)+\beta^{x+n}\left(\beta+\frac{1}{\beta}\right)-\alpha^{n-1} \beta^{x}(1+\alpha \beta)-\alpha^{x} \beta^{n-1}(1+\alpha \beta)\right]$

$=\frac{1}{5}\left[\alpha^{x+n} \sqrt{5}-\beta^{x+n} \sqrt{5}\right]$

$=\frac{\alpha^{x+n}-\beta^{x+n}}{\sqrt{5}}$

$=F(x+1)$

Alternatively, one can use mathematical induction to conclude the $\left(^{*}\right)$.

For $\mathrm{n}=1$, we have;

For $n=1$, we have;

$F(x+1)=f_{I}(x+1)+f_{0} F(x)$

$=f_{1}(x+1)$

$=F(x+1)$

Assuming the equation holds $\forall_{i} \leq n$, we will prove that $\left(^{*}\right)$ is true for $n+1$.

By using the equality

$F(x+2)=F(x)+F(x+1)$, it follows:

$F(x+n+1)=F(x+n)+F(x+n-1)$

$=f_{n} F(x+1)+f_{n-1} F(x)+f_{n-1} F(x+1)+f_{n-2} F(x)$

$=\left(f_{n}+f_{n-1}\right) F(x+1)+\left(f_{n-1}+f_{n-2}\right) F(x)$

$=f_{n+1} F(x+1)+f_{n} F(x)$

\subsection{Observation}

If $f(x)$ is a Fibonacci function, then $\operatorname{Lt}_{x \rightarrow \infty} \frac{f(x+1)}{f(x)}=\frac{1+\sqrt{5}}{2}$

$$
\approx 1.618 \ldots
$$

Proof:

Let L

$=\operatorname{Lt}_{x \rightarrow \infty} \frac{f(x+1)}{f(x)}$

$=\operatorname{Lt}_{x \rightarrow \infty} \frac{f(x)+f(x-1)}{f(x)}$

$=\operatorname{Lt}_{x \rightarrow \infty} 1+\frac{f(x-1)}{f(x)}$ 
$=1+\underset{x \rightarrow \infty}{\operatorname{Lt}} \frac{f(x-1)}{f(x)}$

$=1+\operatorname{Lt}_{x \rightarrow \infty} \frac{f(x)}{f(x+1)}$

$=1+\underset{x \rightarrow \infty}{\operatorname{Lt}}\left(\frac{f(x+1)}{f(x)}\right)^{-1}$

$=1+\left(\underset{x \rightarrow \infty}{\operatorname{Lt}}\left(\frac{f(x+1)}{f(x)}\right)\right)$

$=1+L^{-1}$

Now, by solving, we get;

$$
L=\frac{1 \pm \sqrt{5}}{2}
$$

and we discard the superfluous negative solution $\frac{1-\sqrt{5}}{2}$

i.e., $L=L_{x \rightarrow \infty} \frac{f(x+1)}{f(x)}=\frac{1+\sqrt{5}}{2} \approx 1.618 \ldots$

\section{Remark}

There are several ways to extend the notion of Fibonacci numbers. One of the more common ones is to let $F(x)=\frac{\phi x}{\sqrt{5}}$; where $\phi=\frac{1+\sqrt{5}}{2}$. This gets over the difficulty that $\left(\frac{1-\sqrt{5}}{2}\right)^{x}$ makes no sense for most real values of $x$. Indeed one can in similar way to compute $F(z)$ for complex z.

\section{FIBONACCI SUMMATION}

Let us consider $\sum_{n=0}^{\infty} \frac{F_{n}}{p^{n}}=\frac{p}{p^{2}-p-1},(p>1)$;

Where Fn $=0,1,1,2,3,5,8, \ldots$

Proof: By using Binet's formula;

$F_{n}=\frac{\phi^{n}-(-\phi)^{-n}}{\sqrt{5}}$

By our known geometric series, we have;

$\sum_{n=0}^{\infty} \frac{F_{n}}{p^{n}}=\frac{1}{\sqrt{5}}\left(\frac{1}{1-p \phi}-\frac{1}{1+(p \phi)^{-1}}\right)$

$=\frac{\left(\frac{p\left(\phi^{2}+1\right)}{\sqrt{5}}\right)}{(p-\phi)(p \phi+1)}$

$=\frac{p \phi}{\phi p^{2}-\left(\phi^{2}-1\right) p-\phi}$

$=\frac{p \phi}{\phi p^{2}-\phi p-\phi}$ 
$=\frac{p}{p^{2}-p-1}$

Alternatively,

Let $f(z)=\sum_{n=0}^{\infty} F_{n} Z^{n}$, where $z=\frac{1}{p}$

Wkt

$$
z=f(z)+z^{2} f(z)=\sum_{n=1}^{\infty} F_{n-1} z^{n}+\sum_{n=2}^{\infty} F_{n-2} z^{n}
$$

$$
=F_{0} z+\sum_{n=2}^{\infty}\left(F_{n-2}+F_{n-1}\right) z^{n}
$$

$\left.=F_{0} z+\left[f(z)-F_{0}-F_{1} z\right)\right]$

But, we have; $\mathrm{F}_{0}=0$ and $\mathrm{F}_{1}=1$.

Now, by solving $\mathrm{f}(\mathrm{z})$, we get;

$$
f(z)=\frac{z}{1-z-z^{2}}
$$

As we cited above that $z=\frac{1}{p}$, we see that;

$$
f(z)=\frac{p}{p^{2}-p-1}
$$

\subsection{Observation}

As we stated that $p>1$, for our connivance, I would like to see the limit value at $p=10$.

$\sum_{i=0}^{n} F_{n} z^{n} \quad z=\frac{1}{p}$

$=\frac{(z-1) z^{n+1} F_{n+1}-z^{n+2} F_{n+2}+z}{1-z-z^{2}}$

Here $z<\frac{1-\sqrt{5}}{2}$

Again, by using the Binet's formula;

$$
\begin{aligned}
& \sum_{i=0}^{\infty}=\frac{z}{1-z-z^{2}} \\
& F_{n} z^{n}=\underset{n \rightarrow \infty}{L t} \frac{(z-1) z^{n+1} F_{n+1}-z^{n+2} F_{n+2}+z}{1-z-z^{2}}
\end{aligned}
$$

Now, for $z=1 / 10$ or $p=10$, this limit tend to $10 / 89$.

$(* *)$ can be derived by using Mathematical Induction as by the following Inductive step. [6]

$$
\frac{(z-1) z^{n+1} F_{n+1}-z^{n+2} F_{n+2}+z}{1-z-z^{2}}+z^{n+1} F_{n+1}=\frac{-z^{n+3} F_{n+1}-z^{n+2} F_{n+2}+z}{1-z-z^{2}}
$$




$$
\begin{aligned}
& =\frac{-z^{n+3}\left(F_{n+3}-F_{n+2}\right)-z^{n+2} F_{n+2}+z}{1-z-z^{2}} \\
& =\frac{(z-1) z^{n+2} F_{n+2}-z^{n+3} F_{n+3}+z}{1-z-z^{2}}
\end{aligned}
$$

\section{Acknowledgement}

I am heartily thankful to Mathematician Prof. J. Gopala Krishna and my well-wishers Dr. C.V. Gopinath, Sri N. Nanda Kumar and external supporters R. Decker, Zaboc and A. Nicolas, whose encouragement, guidance and support from the initial to the final level enabled me to develop an understanding of the subject.

\section{References}

[1] http://www.mathsisfun.com/numbers/golden-ratio.html

[2] http://en.wikipedia.org/wiki/Fibonacci_number

[3] http://mathworld.wolfram.com/FibonacciNumber.html

[4] http://sajm.com.nu/sajm2011_1_3_8gandhi.pdf

[5] http://ulcar.uml.edu/ iag/CS/Fibonacci.html

[6] http://www.artofproblemsolving.com/Forum/viewtopic.php?f=36\&p=2084357\#p2084357 